\title{
Early surgical correction of microstomia following Stevens-Johnson syndrome
}

\author{
Sae Hwi Ki ${ }^{1,2}$, \\ Gang Yeon Jo2, \\ Sung Hwan $\mathrm{Ma}^{2}$, \\ Matthew Seung Suk Choi ${ }^{2}$ \\ ${ }^{1}$ Department of Plastic and \\ Reconstructive Surgery, Inha University \\ School of Medicine, Incheon; \\ ${ }^{2}$ Department of Plastic and \\ Reconstructive Surgery, Inha University \\ Hospital, Incheon, Korea
}

Stevens-Johnson syndrome (SJS) is a rare disease in which extensive toxic epidermolysis occurs after medication. Skin and mucous membranes are involved in about 90\% of SJS cases, and webbing of mouth corners (microstomia) may occur when they are affected. Few reports have been issued on microstomia in SJS, and no consensus has been reached regarding treatment methods, timings, or results. We encountered a case of microstomia following SJS after ofloxacin medication in a 22-year-old woman treated by commissuroplasty using a lozenge-shaped excision. We present an appropriate correction method and surgical timing for microstomia following SJS.

Keywords: Microstomia / Mouth mucosa / Steven-Johnson syndrome

\section{INTRODUCTION}

Depending on patient condition, the aim of surgical treatment is to restore normal mouth opening for oral feeding and cosmetic correction. Trauma and postburn contracture are the main causes of microstomia, which makes it difficult to open the mouth fully and results in poor oral hygiene and aesthetic problems. Several surgical methods can be used to treatment the condition depending on its location and extent [1]. In most cases, surgery is usually performed 6 to 12 months after tissue equilibrium has been achieved. During the acute stage of Stevens-Johnson syndrome (SJS), surgeons hesitate to treat microstomia because the condition may recur during the wound healing process due to persistent inflammation.

Microstomia in young socially active patients creates social and oral feeding difficulties, and thus, surgical or conservative treatment should be administered as soon as possible to this patient group. Here, we describe a case of microstomia following SJS in a young woman who was successfully treated at an

\section{Correspondence: Sae Hwi Ki}

Department of Plastic and Reconstructive Surgery, Inha University School of

Medicine, 27 Inhang-ro, Jung-gu, Incheon 22332, Korea

E-mail: mdki1967@gmail.com

Received December 6, 2019 / Revised February 19, 2020 / Accepted March 4, 2020 early stage by commissuroplasty using lozenge-shaped excisions.

\section{CASE REPORT}

A 22-year-old woman was hospitalized in department of allergy due to extensive systemic skin eruption and fever after ofloxacin medication and diagnosed to have SJS. After 4 weeks following medical treatment for SJS, she visited the outpatient clinic of the department of the plastic surgery for the evaluation of microstomia and complained of discomfort when eating, difficulty opening her mouth and cosmetic impairment. Adherence due to mucosal inflammation in bilateral oral commissures had resulted in bilateral oral commissure webbing and a mouth (interlabial) opening of $25 \mathrm{~mm}$. Mucosal inflammation due to SJS was well controlled but skin eruption, mucosal redness, and blisters of lips and in the perioral region were still evident. Two months after visiting our outpatient clinic, erythematous macules, corneal ulceration, oral mucositis, and stomatitis had completely disappeared, there was no evidence of inflammation of oral mucosa, and webbing of the mouth appeared less vascularized, soft, and mobile.

We planned lozenge-shape excisions and closure in a vertical 
direction to lengthen the oral commissure (Fig 1). At 3 months after SJS onset, her maximum mouth opening was $28 \mathrm{~mm}$ between upper and lower lips and the intercommissural distance
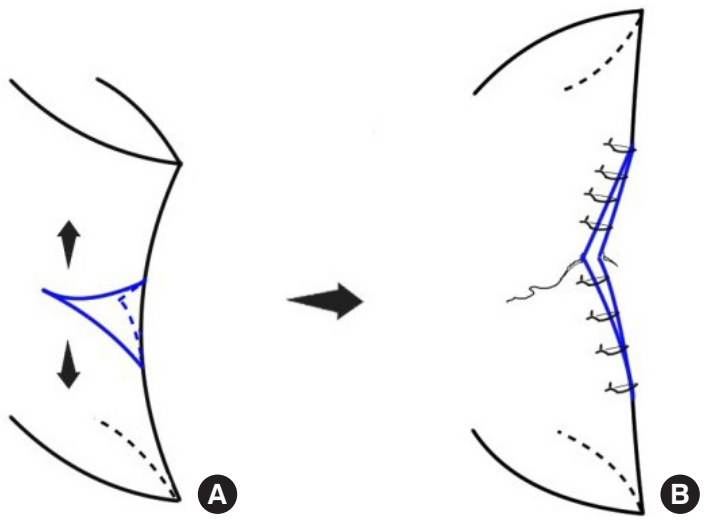

Fig. 1. Operative technique. (A) Drawing of the lozenge shape. (B) Drawing showing simple closure with 6-0 nylon in a vertical direction. was $37 \mathrm{~mm}$ (Fig. 2A). Under local anesthesia, an incision line was designed along both mouth corners (Fig. 2B and C). Lozenge-shaped excisions were made at mouth corners to achieve overcorrection of microstomia, and closure was performed with 6-0 nylon in a vertical direction (Figs. 1B, 3A). After commissuroplasty, an antibiotic ointment was applied, rather than an oral splint. All stitches were removed on postoperative day 7 . At 12 months postoperatively, the mouth webbing had disappeared, the intercommissural distance was $41 \mathrm{~mm}$, and mouth opening was $35 \mathrm{~mm}$ (Fig. 3B). The patient was satisfied with mouth opening, oral feeding, and the cosmetic outcome.

\section{DISCUSSION}

SJS and toxic epidermal necrolysis (TEN) are manifestations of a rare, life-threatening disease, mainly induced by drugs or infection that causes extensive necrosis and epidermis detachment
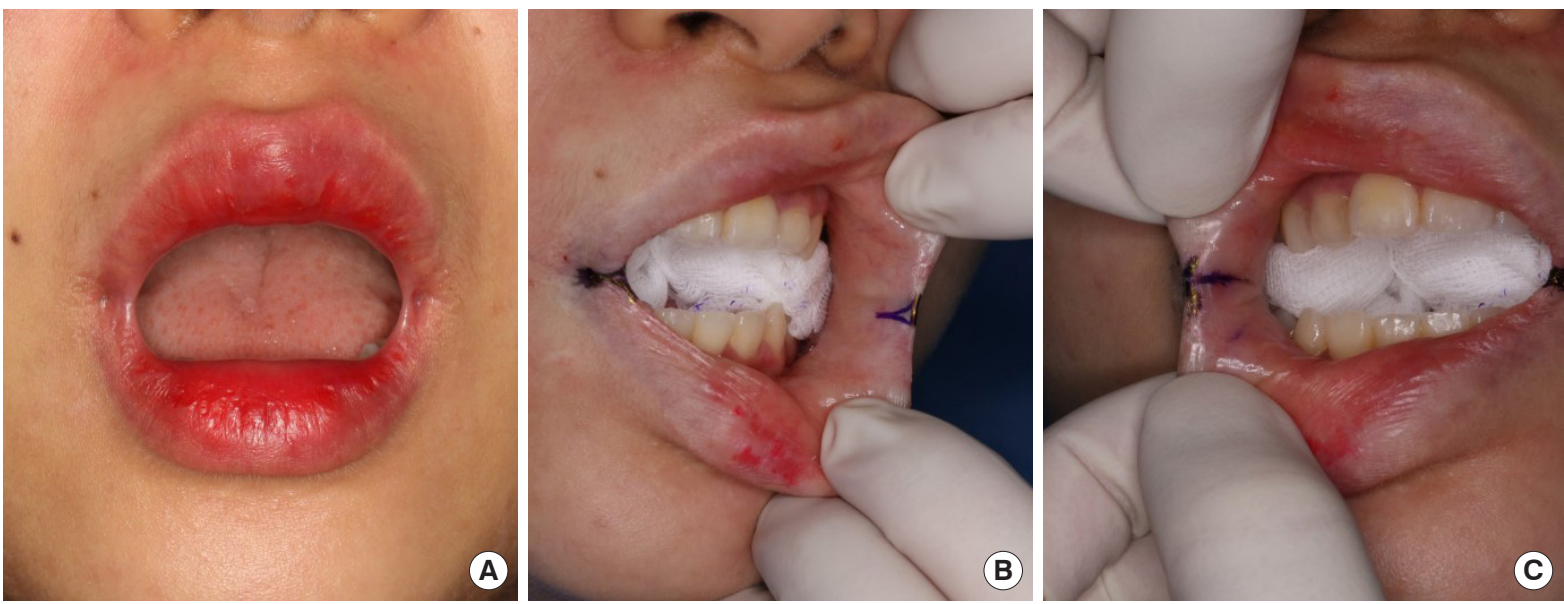

Fig. 2. Preoperative photographs. (A) Lip webbing was observed on bilateral oral commissures 2 months after the disappearance of StevensJohnson syndrome. (B) Preoperative design on the left side oral commissure. (C) Preoperative design on the right-side oral commissure.
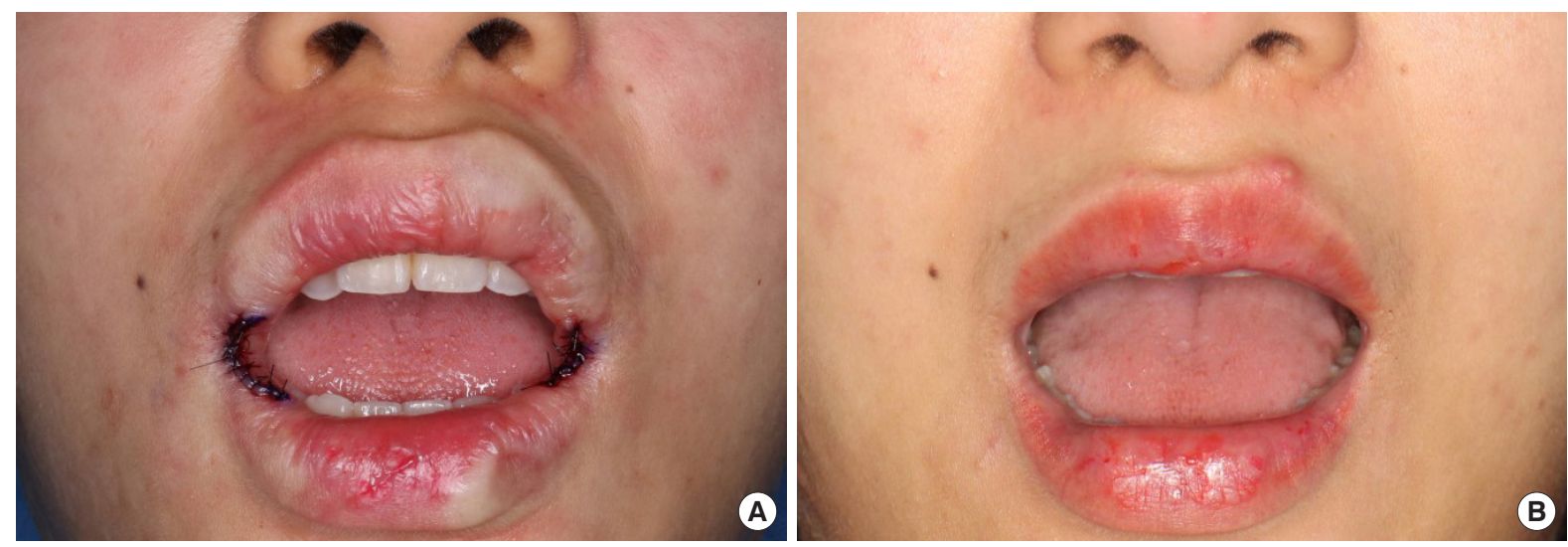

Fig. 3. Postoperative photographs. (A) Immediate postoperative view. (B) After 12 months, the intercommissural distance was 41 mm and mouth opening was $35 \mathrm{~mm}$. 
$[2,3]$. It has been established that T-cells and natural killer cells are involved in SJS, but its etiology is unclear. The combined incidence of SJS and TEN is about two cases per one million people per year [4]. SJS and TEN are differentiated based on percent body surface area (BSA) involvement; the calculation includes skin and mucosa. In SJS, total detachment is less than $10 \%$ of BSA, whereas in TEN this exceeds $30 \%$. In serious cases, the mortality rate of TEN is around 25\% [5]. Mucosal involvement presents in about $90 \%$ of SJS cases and oral mucosa and the vermilion border are predominantly involved. However, microstomia has only been rarely reported to be a sequela of SIS.

The lips are one of the most important functional and cosmetic structures of the face, and narrowing of the oral aperture interferes with oral intake, speech, maintenance of oral hygiene, and dental care. Structural changes caused by microstomia following trauma or burn injury include scar formation in deep tissues involving skin, oral mucosa, and even muscle, and thus, surgical treatment is usually delayed for 6 to 12 months after scar maturation [1]. To treat microstomia, surgeons should excise scar tissue entirely, which unfortunately, results in large, whole tissue defects that include skin and muscle. Triangular scar excision and mucosal Y-V advancement, attributed to Dieffenbach in 1831, reintroduced by Kazanjian and Roopenian [6], and modified by Converse [7], is a one of the most frequently used methods for commissuroplasty [1]. The tissue defects encountered in SJS can be reconstructed using several surgical methods, such as skin and buccal mucosal Y-V advancement, triangular oral mucosal, single rhomboid, trapeze, or fishtail flaps, or skin graft [8-10].

Pathologic changes in microstomia following SJS differ from those observed after burns or trauma, as they are confined to epidermis and mucosa and are not as profound. We were aware that commissuroplasty during the inflammation phase of SJS was likely to cause lip re-adhesion due to ongoing mucosal inflammation. Furthermore, during the correction of microstomia, we overcorrected the pathologic area to cater for some readvancement of webbing at mouth corners. We considered it is important to correct the deformity as soon as possible after the inflammation disappeared, which simplified the procedure and benefited the patient, because untreated microsomia impedes social activities in young patients and causes emotional stress and functional problems.

In the described case, early commissuroplasty was performed 2 months after the disappearance of oral eruption and redness. Functionally, intercommissural distance and mouth opening were improved, and the patient was well satisfied with cosmetic results and the ability to return to normal social life.

\section{NOTES}

\section{Conflict of interest}

No potential conflict of interest relevant to this article was reported.

\section{Ethical approval}

The study was approved by the Institutional Review Board of Inha University Hospital (IRB No. 2019-11-043) and performed in accordance with the principles of the Declaration of Helsinki. Written informed consent was obtained.

\section{Patient consent}

The patients provided written informed consent for the publication and the use of her images.

\section{ORCID}

Sae Hwi Ki https://orcid.org/0000-0001-9194-9681

Gang Yeon Jo https://orcid.org/0000-0003-3951-9830

Sung Hwan Ma https://orcid.org/0000-0002-1956-9016 Matthew Seung Suk Choi https://orcid.org/0000-0002-7849-1861

\section{REFERENCES}

1. Zweifel CJ, Guggenheim M, Jandali AR, Altintas MA, Kunzi W, Giovanoli P. Management of microstomia in adult burn patients revisited. J Plast Reconstr Aesthet Surg 2010;63:e351-7.

2. Mashiko T, Minabe T, Oka A, Ohnishi F. A case of microstomia subsequent to toxic epidermal necrolysis surgically treated by simple technique. Plast Reconstr Surg Glob Open 2013;1:e21.

3. Stern RS, Divito SJ. Stevens-Johnson syndrome and toxic epidermal necrolysis: associations, outcomes, and pathobiologythirty years of progress but still much to be done. J Invest Dermatol 2017;137:1004-8.

4. Rzany B, Mockenhaupt M, Baur S, Schroder W, Stocker U, Mueller J, et al. Epidemiology of erythema exsudativum multiforme majus, Stevens-Johnson syndrome, and toxic epidermal necrolysis in Germany (1990-1992): structure and results of a population-based registry. J Clin Epidemiol 1996;49:769-73.

5. Roujeau JC. Stevens-Johnson syndrome and toxic epidermal necrolysis are severity variants of the same disease which differs from erythema multiforme. J Dermatol 1997;24:726-9.

6. Kazanjian VH, Roopenian A. The treatment of lip deformities resulting from electric burns. Am J Surg 1954;88:884-90.

7. Converse JM. Techniques for the repair of defects of the lips and cheeks. In: Converse JM, editor. Reconstructive plastic surgery. 2nd ed. vol. 3. Philadelphia: Saunders; 1977. p. 1544-94.

8. Johns FR, Sandler NA, Ochs MW. The use of a triangular pedi- 
cle flap for oral commisuroplasty: report of a case. J Oral Maxillofac Surg 1998;56:228-31.

9. Turan A, Tuncel U, Kostakoglu N. The use of single rhomboid flap in reconstruction of microstomia. Burns 2012;38:e24-7.

10. Grishkevich VM. Post-burn microstomia: anatomy and elimination with trapeze-flap plasty. Burns 2011;37:484-9. 